\title{
Decision Focus and Consumer Choice among Assortments
}

\author{
ALEXANDER CHERNEV*
}

\begin{abstract}
This research examines an empirical paradox documented by prior research: when choosing among assortments, consumers opt for the variety offered by larger assortments; however, consumers often are less confident in choices made from larger rather than from smaller assortments. By implying that consumers cannot always accurately predict their need for variety, this preference inconsistency also raises the question of what factors influence consumers' tendency to overestimate their need for the flexibility offered by larger assortments. Building on the view of choice as a hierarchical decision process, this research posits that choice among assortments is a function of consumers' decision focus and, in particular, the degree to which the subsequent task of making a choice from the selected assortment is salient to consumers. The data from four experiments offer converging evidence for the moderating role of decision focus on choice among assortments.
\end{abstract}

$\mathrm{T}$ he findings reported by prior decision research contain an empirical paradox: when choosing among assortments, consumers opt for the variety offered by larger assortments (Broniarczyk, Hoyer, and McAlister 1998; Kahn and Lehmann 1991), yet they often are less confident in choices made from larger rather than smaller assortments (Chernev 2003a, 2003b; Iyengar and Lepper 2000). This preference inconsistency implies that consumers often cannot accurately predict their need for variety and tend to overrate the attractiveness of large assortments. The question then is what drives this tendency to overestimate the need for the flexibility offered by larger assortments.

This research examines how consumers choose among assortments, focusing on factors that are likely to lead to an overestimation of the need for variety. It is proposed that choice among assortments is a function of consumers' decision focus and, in particular, the degree to which the subsequent task of making a choice from the selected assortment is salient to consumers. In this context, it is argued that larger assortments tend to be less preferred by consumers who are readily aware of the potential drawbacks, such as the increased decision complexity, associated with choosing an item from such assortments.

The impact of decision focus on consumer choice among assortments is examined in a series of four experiments,

*Alexander Chernev is associate professor of marketing, Kellogg School of Management, Northwestern University, Evanston, IL 60208 (ach@northwestern.edu). The author thanks the editor, the associate editor, and the three reviewers for their advice and constructive comments.

Dawn Iacobucci served as editor and Stephen Nowlis served as associate editor for this article. which offer converging evidence that consumer choice among assortments is a function of decision focus such that larger assortments are relatively less preferred when the salience of the subsequent product-choice task is high rather than low. The theory motivating these findings, methodology, and data analyses are presented in more detail in the following sections.

\section{THEORETICAL BACKGROUND}

Conventional wisdom suggests that greater choice, almost by definition, benefits consumers. This prediction, prominently featured in the economics literature, builds on the idea that larger assortments offer a greater variety of options that, in turn, increases the probability of a perfect match between a consumer's preferences and the characteristics of the alternatives in the choice set (Baumol and Ide 1956; see Lancaster [1990] for a review). Larger assortments are also likely to be preferred because they allow consumers to maintain flexibility (see Kahn and Lehmann [1991] for a review) so that the variety offered by larger assortments allows them to "keep their options open" in light of uncertainty or ambiguity about future tastes (Kreps 1979; March 1978; see also Kahn, Moore, and Glazer 1987; Kahneman and Snell 1992).

The preference for larger assortments has been further attributed to consumers' inherent tendency to seek variety within or across purchase occasions (McAlister 1982; Ratner, Kahn, and Kahneman 1999), to consumers' preference for options that offer greater decision freedom by imposing fewer constraints on the decision-making process (Reibstein, Youngblood, and Fromkin 1975; see also Brehm and Cohen 1959), as well as to consumers' desire to maintain options 
in the future and avoid the conflict of making a choice in the present (Simonson 1990). Considered together, these findings suggest that when choosing among assortments consumers will opt for the variety offered by larger assortments.

Recent research, however, has questioned the assumption that more variety always benefits consumers. It has been theorized that increasing the size of the choice set can also have adverse consequences on choice because it increases the demand on individuals' cognitive resources, associated with the extra effort required to evaluate the attractiveness of alternatives in the large assortment and potentially leading to a cognitive overload (Hauser and Wernerfelt 1990; Huffman and Kahn 1998; Jacoby, Speller, and Kohn 1974; Malhotra 1982; Scammon 1977; Shugan 1980). It has also been argued that increasing the size of the choice set might confuse consumers, leading to weaker preferences and lower choice probability (Chernev 2003a, 2003b; Dhar 1997; Greenleaf and Lehmann 1995; Iyengar and Lepper 2000; Schwartz et al. 2002). Considered together, these findings suggest that, in the presence of preference uncertainty, choices from large assortments can potentially lead to a lower choice probability and weaker preferences for the selected alternative.

The finding that greater variety can result in choice deferral is inconsistent with the notion that consumers should always prefer larger assortments. This inconsistency raises the question of whether and how consumers "correct" their assortment preferences to account for the potential adverse impact of larger assortments. In this context, this research argues that consumers' tendency to overestimate their future need for variety is moderated by their decision focus and, in particular, by the degree to which the subsequent task of choosing an option from the selected assortment is salient to consumers.

To address the paradox that greater variety is preferred even when it leads to weaker preferences and greater choice deferral, this research adopts a conceptual framework that views choice as a hierarchical decision process that comprises two different stages: selecting an assortment and, subsequently, selecting an option from that assortment (Kahn and Lehmann 1991; Kahn, Moore, and Glazer 1987; Sood, Rottenstreich, and Brenner 2004; see also Bettman and Park 1980; Payne 1976; Tversky and Sattath 1979). In this context, the observed discrepancy in consumer preferences when choosing an assortment and when choosing an item from the selected assortment can be attributed to the nature of the consumer decision process and, in particular, to whether these two stages of the overall decision are considered jointly or separately. Thus, increasing the salience of the product-choice task should also increase the probability that consumers will view the assortment choice as a joint two-stage decision (instead of two independent choices) and will, therefore, select the assortment that allows them to optimize the choice of an item from that assortment. In this context, if the choice of an assortment and the subsequent product selection are viewed as two independent decisions, then choosing the larger assortment is likely to be perceived as the optimal strategy. If, however, both decisions are considered jointly, the choice of an assortment is likely to be influenced by a consumer's desire to optimize the subsequent choice as well. As a result, when consumers believe that choosing a product from the larger assortment is likely to have substantial drawbacks, such as the increased decision difficulty, the probability of choosing that assortment is likely to decrease.

To illustrate, consider two consumers who are choosing among assortments that vary in size, such that one consumer is focused only on choosing among the two available assortments, whereas the other is also focused on selecting the optimal product from the chosen assortment. In this context, it is argued that this different decision focus is likely to activate different decision strategies. A consumer who is focused only on the choice among assortments will be more likely to display a preference for larger assortments because of uncertainty about future preferences (Kreps 1979) and a desire to put off the effort of making trade-offs into the future (Simonson 1990). In contrast, a consumer who focuses on the subsequent task of selecting an option from the chosen assortment will be less likely to display a preference for larger assortments because of the anticipated difficulty of making a choice from such assortments (Chernev 2003b).

To summarize, this research argues that consumer choice among assortments is guided by two potentially conflicting goals. When choosing among assortments, the goal is to maximize decision flexibility and "hedge" against future preference uncertainty. In contrast, when choosing an option from a given assortment, the goal is to simplify the decision process and select the best available alternative. In this context, this research argues that the preference for larger assortments is likely to be a function of consumers' decision focus, such that larger assortments will be more preferred (relative to smaller assortments) when the choice among assortments is considered independently of the productchoice task than when the product-selection and the assortment-selection tasks are considered together. This prediction is tested in a series of four experiments described in more detail next.

\section{EXPERIMENT 1}

The goal of experiment 1 is to test empirically the proposition that consumer choice among assortments is a function of the salience of the subsequent product-choice task, such that larger assortments are relatively less preferred when the salience of the subsequent product choice is high than when it is low.

\section{Method}

Respondents, 111 Northwestern University undergraduates, were asked to imagine that they had to purchase snacks for two teammates with unknown preferences. Respondents were told that they could choose between two vending ma- 
chines: one offering six different snacks (the most popular snack from each of the following categories: chocolate bars, snack crackers, chips, health food bars, nonchocolate candy, and sugarless candy) and one offering 36 different snacks (six snacks from each of the above categories). The use of snacks as experimental stimuli and the six subcategories were suggested by prior research (Kahn and Lehmann 1991; Simonson 1990).

Respondents were randomly assigned to two conditions. Respondents in the assortment-focus condition were told that they would be asked to explain their choice of a vending machine to their teammates. In contrast, respondents in the product-focus condition were told that they would be asked to account for their choice of snacks. This manipulation was derived from the need-for-justification paradigm introduced in prior research (Simonson 1989; Simonson and Nowlis 2000).

Following the decision-focus manipulation, respondents in both conditions were asked to select one of the two vending machines and provide the rationale for their decision. The experiment was conducted online; respondents were recruited by e-mail and paid $\$ 5$ for participating.

\section{Results}

This research argued that the choice among assortments is a function of the salience of the product-choice task, so that smaller assortments are more preferred when the salience of the subsequent product choice is high rather than when it is low. A test of this proposition is given by comparing the dispersion of choice shares of the two vending machines across the two experimental conditions. The data show that only $1.8 \%$ of the respondents in the assortmentfocus condition selected the vending machine offering the smaller assortment; the rest preferred the vending machine with the greater assortment $(N=57)$. In contrast, the smaller assortment was preferred by $35.2 \%$ of the respondents in the product-focus condition while the rest preferred the larger assortment $(N=54)$.

The significance of the above data pattern was tested by examining respondents' choice of an assortment as a function of their decision focus. The analysis shows that the dispersion of choice shares of the smaller assortment across the two justification conditions was significant $\left(\chi^{2}(1)=\right.$ $10.61, p<.005)$. The finding that respondents in the product-focus condition were more likely to prefer smaller assortments than respondents in the assortment-focus condition is consistent with the experimental predictions.

Analysis of respondents' self-reported choice reasons (e.g., Ericsson and Simon 1980) offers further insight into the decision processes underlying their choices. Respondents' reasons were classified into four categories: (1) variety, (2) selection, (3) easier to decide, and (4) other. The variety category included responses such as "more variety to choose from" and "more likely to find what I want." The selection category included references to the fact that the smaller selection included only the most popular options, such as "How can I go wrong if I choose the most popular snack?" and "I feel safer to get the products considered to be "popular." The easier-to-decide category included reasons such as "makes the choice easier because I had limited number of snacks to choose from" and "less selection so less confusing." Responses that could not be classified in any of the foregoing categories were listed in the "other" category.

Of the respondents in the assortment-focus condition, $94.6 \%$ indicated variety as the primary reason for their selection, and none of these respondents selected ease of the decision $(N=56)$. In contrast, among the respondents in the product-focus condition, $20.8 \%$ explicitly indicated ease of the decision as their primary reason for choice, $11.3 \%$ indicated selection as their reason for choice, and $54.7 \%$ selected variety as their reason for choice $(N=53)$. The corresponding analysis shows that the number of respondents who used the easier-to-decide argument was significantly greater in the product-focus than the assortment focus conditions $\left(\chi^{2}(1)=12.93, p<.001\right)$. Furthermore, for respondents who explicitly identified ease of decision as a primary choice reason, decision focus had a significant impact on choice among assortments as well $\left(\chi^{2}(1)=7.73\right.$, $p<.01)$, lending further support to the experimental predictions.

\section{Discussion}

The data furnished by this experiment support the proposition that choice among assortments is a function of the salience of the subsequent product-choice task and that smaller assortments are likely to be more preferred when the subsequent product-choice task is salient to consumers. To test the proposition that choice among assortments is a function of the salience of the subsequent product task, this experiment employed a justification-based manipulation to vary respondents' decision focus. An alternative strategy to manipulate decision focus is to use respondents' prior experience in making choices from assortments that vary in size. In this context, the salience of the product-choice task is likely to be greater for individuals who have recently made product choices from large assortments and are, therefore, more likely to be readily aware of the potential drawbacks of choosing from larger assortments. This proposition is tested in the following experiment.

\section{EXPERIMENT 2}

The goal of this experiment is to test the proposition that in the presence of preference uncertainty the salience of the product-choice task will influence consumer choice among assortments, leading to a decrease in the share of the larger assortment. Unlike experiment 1 , in which this proposition was tested by using a choice-justification manipulation, experiment 2 relies on prior experience to manipulate individuals' decision focus. In this context, the impact of decision focus on choice among assortments is tested by introducing a learning task in which respondents had to either make a choice from a relatively large assortment 
(product-choice task) or simply evaluate the assortment without making a choice (product-evaluation task). This learning task was followed by an assortment-choice task in which all respondents were asked to choose between a larger and a smaller assortment. In this context, it is argued that respondents in the product-choice task who had to choose one of the alternatives were more likely to experience the decision difficulty associated with larger assortments and, as a result, should be less likely to choose the larger assortment in the subsequent decision task. In contrast, respondents who were not explicitly asked to make a choice in the learning task and did not experience the drawbacks associated with choosing from the larger assortment should be more likely (relative to those in the product-choice task) to choose the larger assortment in the subsequent decision task.

\section{Method}

Respondents, 138 Northwestern University undergraduates, were randomly assigned to one of the two experimental conditions. Respondents in the product-choice condition were asked to evaluate a relatively large selection of Godiva chocolates and were asked to select their most preferred chocolate. The selection contained 80 different pieces and was organized in eight rows of 10 items each. Each of the individual chocolates was represented by a picture and a description (e.g., honey roasted almond truffle). The product selection was suggested by prior assortment research (Chernev 2003a, 2003b; Iyengar and Lepper 2000), and the product descriptions were obtained from Godiva Chocolatier.

Respondents were asked to choose their most preferred chocolate and then indicate how difficult it was for them to make a choice from the above selection (nine-point scale: 1 = very easy; $9=$ very difficult), how satisfied they were with the above assortment (nine-point scale: $1=$ very satisfied; $9=$ not satisfied at all), and how they would rate the selection offered by the assortment (ninepoint scale: 1 = very limited; 9 = very large). The learning task for the respondents in the product-evaluation condition was identical except that they were not asked to make a choice and, consequently, were not asked the choice-difficulty question.

Following the learning task, respondents in both conditions were asked to imagine that they had received a gift certificate for a box of Ghirardelli chocolates and could redeem the certificate in one of the two stores specializing in chocolates. They were further told that one of the stores (store A) carries a selection of 24 chocolates, whereas the other store (store B) carries a selection of 88 chocolates. To avoid any potential assortment-quality inferences, both stores were said to have identical customer satisfaction ratings (five stars). Respondents were then asked to select the store in which to buy their most preferred chocolate. The experiment was conducted online; respondents were recruited by e-mail and paid $\$ 5$ for participating.

\section{Results}

It was theorized that choice among assortments is a function of the salience of the product-choice task, so that larger assortments would be more preferred by respondents in the product-evaluation condition than by those in the productchoice condition. The data show that only $2.2 \%$ of the respondents $(N=46)$ in the product-evaluation condition selected the smaller assortment in the subsequent task compared to $16.3 \%$ of the respondents $(N=92)$ in the product-choice task. The significance of this data pattern was tested by examining respondents' choice of an assortment as a function of the nature of the decision task. The analysis shows that the difference in the impact of the nature of the learning task (product choice vs. product evaluation) on choice among assortments was significant $\left(\chi^{2}(1)=\right.$ $4.28, p<.05)$, a finding consistent with the experimental predictions.

Further support for the theoretical predictions advanced in this research can be obtained by evaluating whether and how respondents' self-reported decision difficulty moderates their choice among assortments. Indeed, the theory advanced in this research argues that consumers are likely to avoid larger assortments because of the complexity associated with making choices from such assortments. In this context, one could argue that respondents who perceived the initial product-choice task to be relatively difficult would be less likely to choose the larger assortment in the subsequent task compared to respondents who perceived the initial product-choice task to be relatively easy.

To test the impact of decision difficulty on choice among assortments, respondents were divided into two groups: those who rated the choice task to be relatively easy (ratings 1-4 on the nine-point scale) formed the easy-choice group, whereas those who perceived the product-choice task to be relatively difficult (ratings 6-9 on the nine-point scale) formed the difficult-choice group. Based on this split, there were 40 respondents in the easy-choice group and 36 in the difficult-choice group. Consistent with the theoretical predictions, the data show that the smaller assortment was preferred by $28.2 \%$ of respondents in the difficult-choice group but only by $7.5 \%$ of respondents in the easy-choice group $\left(\chi^{2}(1)=5.11, p<.05\right)$.

\section{Discussion}

The data furnished by experiment 2 lend support to the prediction that the salience of the product-choice task is likely to influence consumer choice among assortments, leading to a decrease in the share of the larger assortment in cases when the complexity of the product-choice task is salient to consumers. Building on the findings of experiment 1 , in which decision focus was manipulated by varying the nature of the justification task (assortment justification vs. product justification), this experiment varied decision focus by using a learning task to make salient the decision complexity associated with choices from large assortments. In this context, the data show that respondents who were given 
the complexity-learning task were less likely (compared to those who were not given the complexity-learning task) to subsequently select the larger assortment. Moreover, the data show that this effect was more pronounced for respondents who perceived the learning task to be relatively difficult than for those who perceived the learning task to be relatively easy.

In this experiment, the decision focus was manipulated by varying the salience of the decision difficulty associated with choosing from larger assortments. An alternative strategy to manipulate decision focus is to vary the temporal distance between the choice of an assortment and the choice of a product from the selected assortment. Thus, when these two choices are temporally separated, they are likely to be considered in isolation, and consumers are likely to maximize the utility of each decision independently. In contrast, a compression of the perceived time interval between the two decisions is likely to increase the salience of the productchoice task (Read and Loewenstein 1995), making consumers treat assortment selection and product choice as a single decision. In this context, it is proposed that as the temporal distance between the assortment selection and product choice decreases, consumers will be more likely to forgo the flexibility offered by the larger assortment and choose the smaller assortment. This prediction is empirically examined in the following experiment.

\section{EXPERIMENT 3}

The goal of this experiment is to test the proposition that the impact of decision focus on choice among assortments can be attributed to the hierarchical nature of the choice process, such that consumers' selection of an assortment is often independent from their choice of a product from the selected assortment. This proposition is tested by manipulating the temporal proximity of the two stages of the choice process, assortment choice and product choice. In this context, the goal is to show that decreasing the temporal distance between the assortment selection and product choice is likely to be associated with an increase in the preference for the smaller assortment.

\section{Method}

Respondents, 88 Northwestern University undergraduates, were asked to imagine that they were buying a pen and had two selections to choose from. The first selection (store A) included 12 Waterman pens, and the second selection (store B) offered a selection of 60 Waterman pens. Pens in both selections were described by a picture and descriptive text (e.g., Waterman Hemisphere Collection). Options were organized in two columns and six rows for the smaller assortment and two columns and 30 rows for the larger assortment. Both assortments were presented on the same page: smaller assortment first, followed by the larger assortment, followed by the dependent measure questions.

Respondents were randomly assigned to two conditions.
Respondents in the immediate product-choice condition were told that their goal was to select a store and then select a pen from that store. In contrast, respondents in the delayed product-choice condition were told that they had to make their store selection immediately but would make their purchase on a separate occasion next month. Respondents in both conditions were told that after making the purchase they might be asked to explain their choice of a pen. Respondents were then asked to select one of the two stores and to indicate their satisfaction with each of the two assortments (nine-point scale: $1=$ very unsatisfied; $9=$ very satisfied). The experiment was conducted online; respondents were recruited by e-mail and paid $\$ 5$ for participating.

\section{Results}

This research argued that consumer choice among assortments is a function of the temporal proximity of the assortment-choice and product-choice tasks, such that consumers will be less likely to choose the larger assortment when it is immediately followed by a product-choice task than when the product-choice task is temporally separated from the choice of an assortment. A test of this prediction is given by examining the dispersion of respondents' assortment choices as a function of the temporal proximity of the product-choice task. The data show that $19.2 \%$ of the respondents in the immediate product-choice condition $(N=36)$ selected the smaller assortment. In contrast, of those in the delayed product-choice condition $(N=52)$ only $2.8 \%$ selected the smaller assortment. Statistical analysis of these data shows that the difference in the likelihood of choosing an assortment as a function of the temporal distance of the product-choice task was significant $\left(\chi^{2}(1)=\right.$ $3.90, p<.05)$, a finding consistent with the experimental predictions.

Further evidence in support of the experimental predictions can be obtained by examining respondents' satisfaction with the two available assortments as a function of the temporal proximity of the product-choice task. The theory advanced in this research predicts that the potential disadvantages associated with making choices from large assortments will be more salient to consumers who are simultaneously considering the choice of an assortment and the choice of a particular product from the selected assortment than to consumers for whom these two choices are temporally separated. The data show that, compared to those in the delayed product-choice condition, respondents in the immediate product-choice condition were more satisfied with the smaller assortment ( $M=6.48$ vs. $M=6.11)$ and less satisfied with the larger assortment $(M=7.38$ vs. $M=7.72)$. Statistical analysis of these data shows that the impact of temporal distance of the product-choice task on the difference between respondents' satisfaction with the large versus small assortment was significant $(M=1.61$ vs. $M=.90$; $F(1,86)=4.29 ; p<.05)$, a finding consistent with the experimental predictions. 


\section{Discussion}

The data from this experiment document that decreasing the temporal distance between the assortment selection and product choice is likely to be associated with an increase in the preference for smaller assortments. In this context, this experiment documents that respondents were less likely to select the larger assortment in a scenario when the choice of an assortment was immediately followed by a productchoice task than when these two tasks were temporally separated. This finding is consistent with the theorizing that the impact of decision focus on choice among assortments can be attributed to the hierarchical nature of the choice process, such that consumers' selection of an assortment is often independent from their choice of a product from the selected assortment.

The data furnished by the first three experiments show that making salient the complexity of the subsequent product-choice task is likely to decrease the probability of choosing the larger assortment. This finding however, is based on the assumption that choosing from larger assortments is, in fact, associated with more complex decisions. Indeed, it was argued that increasing the size of the assortment tends to complicate the decision process and decrease the overall probability of purchase - an effect attributed to the increased difficulty of identifying the alternative that best matches an individual's ideal point. In this context, the presence of a certain level of decision complexity associated with the larger but not the smaller assortment is a necessary condition for the observed data pattern. Following this line of reasoning, one could argue that in cases when the choice from larger assortments is straightforward and does not involve complex information processing, the impact of decision focus on choice among assortments is likely to be less pronounced.

The above argument suggests that the impact of decision focus on choice among assortments can be further tested by manipulating the difficulty of choosing from these assortments. One approach to manipulating decision difficulty is to vary the composition of the choice set. Thus, prior research has proposed that when consumers are uncertain in their preferences, the dominant relationships among the alternatives in the choice set can provide a sufficient reason for choice (Simonson 1989; Simonson and Tversky 1992; Tversky, Sattath, and Slovic 1988). In this context, it was proposed that when consumers are provided with a compelling decision rule, such as relative dominance, larger assortments are less likely to confuse consumers than when such a decision rule is not available.

Building on this rationale, this research posits that the influence of the decision focus on the choice among assortments is moderated by the dominance relationships among options comprising these assortments. In particular, the increase in the relative share of the smaller assortment associated with the increased salience of the product-choice task will be greater when none of the options in the choice set dominates the others than when a dominant alternative is present. This proposition is empirically tested in the following experiment.

\section{EXPERIMENT 4}

The goal of this experiment is to test the prediction that the impact of decision focus on the choice among assortments is moderated by the dominance relationships among options in the set, such that the relative preference for the smaller assortment associated with product-focused decisions will be greater when none of the options in the choice set dominates the others than when a dominant alternative is present. In this context, the dominance relationships among choice options were varied using two alternative manipulations: (1) by varying options' performance on the most important attribute and (2) by varying respondents' familiarity with the choice options. These two manipulations correspond to the two different approaches to conceptualizing dominance: local dominance, derived from the readily available information about the choice alternatives, and background dominance, derived from information about choice alternatives from past experience (Simonson and Tversky 1992). In this context, local dominance was manipulated by featuring one of the options as dominant on the most important attribute - a manipulation consistent with the prominence principle, stating that dominance on the primary attribute offers a compelling reason for choice (Tversky, Sattath, and Slovic 1988). In contrast, the background dominance manipulation varied respondents' familiarity with the options in the set-a proposition consistent with prior research documenting that familiar objects are more likely to be chosen than unfamiliar objects (Alba and Hutchinson 1987; Hawkins and Hoch 1992). The experimental method and the data are given in more detail in the following sections.

\section{Method}

One hundred and eighty-eight Northwestern University undergraduates were assigned to the conditions of a 2 (decision focus: assortment vs. product) $\times 3$ (set dominance: none vs. local vs. background) between-subjects full factorial design. Respondents were asked to imagine that they had won a free trip to Bermuda from the local radio station and had a choice of two travel agencies to arrange their trip: Bermuda Sun Travel and Bermuda Vacations. Both agencies were said to have been recommended by friends and to offer similar packages (direct flights to Bermuda, transfers to and from the hotel, ocean-view suite, etc.). The only difference was the number of hotels to choose from: Bermuda Sun Travel offered a choice of 24 five-star hotels and Bermuda Vacations offered a choice of six five-star hotels.

This instruction was followed by the descriptions of the resorts offered by each of the two agencies. All resorts were described by name, hotel amenities, and room features. Hotel amenities and room features were identical for all resorts and included a pool, restaurant, bar, and spa (hotel amenities) and balcony, Jacuzzi, TV, phone, and daily maid service 
(room features). Resorts in the smaller assortment (Bermuda Vacations) were a subset of the resorts in the larger assortment (Bermuda Sun Travel). Resorts offered by each agency were presented side by side, and their description was followed by the dependent-variable questions.

Decision focus was manipulated by informing respondents that as a part of the free-trip promotion they would be asked to explain to radio listeners either their choice of a travel agency (assortment-focus condition) or their choice of a resort (product-focus condition). This decision-focus manipulation was conceptually similar to the one used in the first experiment.

The complexity of the choice set was manipulated by varying the type of relationships among its options. In the background-dominance conditions, decision complexity was manipulated by varying respondents' familiarity with the options, so that one of the alternatives was familiar to respondents (Ritz-Carlton) and all other options were relatively unknown (e.g., Astwood, Granaway, Rosedon, Sandpiper, Sonesta, and Clearview). In contrast, in the local-dominance conditions, none of the choice alternatives was familiar; instead, the dominance was manipulated by varying options' performance on a key attribute (hotel location), so that one of the alternatives (beachfront resort) dominated the others (located near the beach).

The dependent variable was respondents' choice among assortments and their satisfaction with the assortment offered by each of the travel agencies (nine-point scale: $1=$ not satisfied at all; $9=$ very satisfied). For the purposes of manipulation check, at the end of the experiment respondents in the local-dominance condition were asked to indicate their preference for a beachfront or off-the-beach resort. The experiment was conducted online; respondents were recruited by e-mail and paid $\$ 5$ for participating.

\section{Results}

This research posits that the effect of decision focus on choice among assortments is moderated by the relative attractiveness of options comprising these assortments, so that an increase in the relative share of the smaller assortment associated with an increase in the salience of the productchoice task will be less pronounced in the presence of a dominant alternative. This finding was attributed to the fact that the presence of a dominant option is likely to decrease the complexity of the decision, which in turn moderates the impact of decision focus on choice among assortments.

One hundred and eighty-six observations were used in the data analyses (two observations were deleted after the manipulation check revealed that these respondents preferred off-the-beach resort locations). The choice share data, summarized in table 1 , show that the impact of decision focus on the choice among assortments is greater in the absence of a dominant alternative than when a dominant alternative is present. To illustrate, in the absence of a dominant option, only $2 \%$ of the respondents selected the smaller assortment when expecting to have to explain their choice of a travel agency (assortment-focus condition), compared
TABLE 1

THE SHARE OF THE SMALLER ASSORTMENT AS A FUNCTION OF DECISION FOCUS AND SET DOMINANCE (EXPERIMENT 4)

\begin{tabular}{lcc}
\hline \hline & \multicolumn{2}{c}{ Decision focus } \\
\cline { 2 - 3 } Dominant alternative & $\begin{array}{c}\text { Assortment } \\
(\%)\end{array}$ & $\begin{array}{c}\text { Product } \\
(\%)\end{array}$ \\
\hline $\begin{array}{l}\text { Not present } \\
\begin{array}{l}\text { Local dominance } \\
\text { (primary attribute) }\end{array}\end{array}$ & $2.4(41)$ & $34.1(44)$ \\
$\begin{array}{l}\text { Background dominance } \\
\text { (familiar brand) }\end{array}$ & $7.7(26)$ & $3.8(26)$ \\
\hline NOTE-Cell sizes are given in parentheses. & $0(23)$ \\
\hline
\end{tabular}

NotE.-Cell sizes are given in parentheses.

to $34 \%$ who selected the smaller assortment when expecting to have to justify their choice of a resort (product-focus condition). In contrast, the corresponding choice shares were $8 \%$ and $4 \%$ when an option superior on a prominent attribute was present (local-dominance condition) and $4 \%$ and $0 \%$ when an attractive familiar brand was present (backgrounddominance condition).

Statistical analysis of these data shows that the impact of decision focus on the choice among assortments was indeed a function of set dominance $\left(\chi^{2}(1)=6.75, p<.001\right)$. Consistent with the experimental predictions, this effect was significant for both local and background dominance conditions $\left(\chi^{2}(1)=5.23, p<.05\right.$ and $\left.\chi^{2}(1)=5.78, p<.05\right)$. Further analysis shows that, in the absence of a dominant alternative, the impact of decision focus was significant $\left(\chi^{2}(1)=8.15, p<.005\right)$, a finding that further supports the proposition that choice among assortments is a function of the salience of the subsequent product-choice task, such that larger assortments are less preferred when the salience of the subsequent product choice is high rather than when it is low.

In addition to the choice share analysis, the experimental predictions can be further tested by examining the dispersion of respondents' satisfaction with the available assortments across the experimental conditions. Because each respondent was asked to indicate his/her satisfaction with both the small and the large assortment, a relative satisfaction measure was calculated for each individual. In this context, it was proposed that (1) respondents would be more satisfied (in relative terms) with the smaller assortment when the product-choice task was salient and (2) that this effect would be less pronounced in the presence of a dominant alternative.

The mean differences in attractiveness of the larger and the smaller sets across the experimental conditions are given in table 2. The data show that the difference in the relative attractiveness of the larger and the smaller assortments was greater when none of the options dominated the others $(M=2.85$ vs. $M=1.32)$ than when a dominant option was present ( $M=1.65$ vs. $M=1.61$ and $M=2.27$ vs. $M=2.13)$. Analysis of these data shows that the set dominance significantly moderated the impact of the decision focus on respondents' relative satisfaction with the available 
TABLE 2

THE MEAN DIFFERENCES IN ATTRACTIVENESS OF LARGE AND SMALL ASSORTMENTS AS A FUNCTION OF DECISION FOCUS AND SET DOMINANCE (EXPERIMENT 4)

\begin{tabular}{llc}
\hline \hline & \multicolumn{2}{c}{ Decision focus } \\
\cline { 2 - 3 } Dominant alternative & Assortment & Product \\
\hline $\begin{array}{l}\text { Not present } \\
\begin{array}{l}\text { Local dominance } \\
\text { (primary attribute) }\end{array}\end{array}$ & $2.85(1.85)$ & $1.32(2.00)$ \\
$\begin{array}{l}\text { Background dominance } \\
\text { (familiar brand) }\end{array}$ & $1.65(1.72)$ & $1.61(1.63)$ \\
\hline
\end{tabular}

NOTE.-Standard deviations are given in parentheses. Cell sizes correspond to those given in table 1 .

assortments $(F(1,182)=4.56, p<.01$ for the backgrounddominance condition and $F(1,182)=5.45, p<.01$ for the local-dominance condition). These findings lend support to the proposition that decision focus moderates the choice among assortments and that this effect is a function of the dominance relationship among the alternatives in the choice sets.

\section{Discussion}

The data from experiment 4 show that the impact of the decision focus on choice among assortments is a function of decision difficulty and, in particular, the dominance relationships among options comprising these assortments. In particular, the increase in the relative share of the smaller assortment associated with the increased salience of the product-choice task, reported in experiments 1-3, was found to be significant only when none of the options in the choice sets dominated the others; in the presence of a dominant alternative the decision-focus effect was absent. This finding was tested using two different conceptualizations of dominance relationships in the choice set: local dominance, derived exclusively from the attribute information offered by the decision task, and background dominance, based on consumers' prior experience with the choice alternatives. The convergence of the data across these two conditions lends further support to the proposition that the presence of decision complexity associated with choosing from larger assortments is a necessary condition for the observed decision focus effect.

\section{GENERAL DISCUSSION}

This research examined the role of decision focus as a factor moderating consumer choice among assortments. Building on the view of assortment choice as a hierarchical decision process in which consumers first select an assortment and then choose an option from the selected assortment, it was argued that consumers who focus only on the first stage of this process (selecting an assortment) are more likely to display a preference for the decision flexibility offered by larger assortments. In contrast, consumers who focus on the second stage of the choice process (choosing a product from the readily selected assortment) are more likely to display a preference for the decision simplicity often afforded by smaller assortments.

The moderating role of decision focus on the choice among assortments is documented in a series of four experiments, which report converging empirical evidence for the theoretical predictions. In particular, the influence of decision focus on choice among assortments is documented (1) by varying the nature of the justification task (assortment justification vs. product justification), (2) by making salient the drawbacks of choosing from a larger assortment, and (3) by manipulating the temporal distance between the assortment selection and product choice. The data obtained by these manipulations offer converging evidence that choice among assortments is a function of consumers' decision focus, such that the preference for larger assortments tends to be greater when the decision focus is on choosing the assortment than when the focus is on making a choice from the readily selected assortment.

In addition to documenting the impact of decision focus on choice among assortments, this research also identifies some of its boundary conditions and shows that the impact of decision focus is a function of decision difficulty. In particular, the decrease in the choice share of the larger assortment in a product-focused (vis-à-vis assortment-focused) context was found to be less pronounced in the presence of a simple decision criterion, such as the presence of a dominant alternative. This finding is consistent with the notion that the decrease in the relative preference for larger assortments is contingent on the complexity of the choice task, such that the presence of decision difficulty associated with choosing from the larger assortment is an important precondition for observing the decision-focus effect.

The findings reported in this research are subject to certain limitations, many of which also offer directions for further investigation. Thus, although all four experiments reported in this article show an increase in the relative share of the smaller assortment as a function of decision focus, most of the respondents nevertheless preferred the larger assortment. This observation underscores the importance of identifying additional factors moderating consumer choice among assortments. One factor that is likely to influence consumer preference for smaller versus larger assortments is the overall attractiveness of these assortments (Chernev and Hamilton 2005). Thus, it is possible that consumers are more likely to prefer smaller sets when the attractiveness of options comprising these sets is high rather than when it is low. To illustrate, the preference for smaller assortments is likely to be greater when choosing among sets containing a selection comprising only the most popular options than when choosing among sets in which the relative attractiveness of the options is unknown. Investigating the impact of the composition of the assortment on consumer choice among assortments is a promising venue for future research.

Another issue not explicitly addressed in this research concerns decisions involving the purchase of multiple items 
and, in particular, how the relationship between the size of the assortment and the number of to-be-purchased items is likely to influence consumer choice among assortments (Chernev 2005). In this context, one can argue that a match between the assortment size and the number of to-be-purchased items allows consumers to simplify choice and, thus, avoid the need to trade off the benefits and costs of individual choice alternatives. To illustrate, by choosing an assortment comprising five alternatives, a consumer who is purchasing items for five different individuals can make an easy choice from that assortment by simply purchasing one item for each individual. In the same vein, when purchasing items to be consumed on five different occasions, a consumer might prefer an assortment comprising five items because selecting the five-option assortment offers an inherent product-allocation strategy: a different item each day. Investigating the impact of the purchase goals on choice among assortments is a promising area for further investigation.

Overall, the findings reported in this article contribute to the literature by resolving the empirical paradox implied by prior findings, whereby larger assortments, although more frequently chosen than smaller assortments, often tend to yield less confident choices. The decision-focus theory advanced in this research implies that the observed preference inconsistency can be attributed to a shift in consumer decision goals, from maximizing decision flexibility, when choosing among assortments, to minimizing decision complexity and reaching a readily justifiable decision, when choosing a product from the readily selected assortment. In this context, the research reported in this article demonstrates that by varying the decision focus, it is possible to systematically vary consumers' choice among assortments.

\section{REFERENCES}

Alba, Joseph W. and J. Wesley Hutchinson (1987), "Dimensions of Consumer Expertise," Journal of Consumer Research, 13 (March), 411-55.

Baumol, William J. and Edward A. Ide (1956), "Variety in Retailing," Management Science, 3 (1), 93-101.

Bettman, James R. and C. Whan Park (1980), "Effects of Prior Knowledge and Experience and Phase of the Choice Process on Consumer Decision Processes: A Protocol Analysis," Journal of Consumer Research, 7 (December), 234-48.

Brehm, Jack W. and Arthur R. Cohen (1959), "Reevaluation of Choice Alternatives as a Function of Their Number and Qualitative Similarity," Journal of Abnormal and Social Psychology, 58, 373-78.

Broniarczyk, Susan M., Wayne D. Hoyer, and Leigh McAlister (1998), "Consumers' Perceptions of the Assortment Offered in a Grocery Category: The Impact of Item Reduction," Journal of Marketing Research, 35 (September), 166-76.

Chernev, Alexander (2003a), "Product Assortment and Individual Decision Processes," Journal of Personality and Social Psychology, 85 (July), 151-62.

(2003b), "When More Is Less and Less Is More: The Role of Ideal Point Availability and Assortment in Consumer
Choice," Journal of Consumer Research, 30 (September), $170-83$.

(2005), "Goal Compatibility in Assortment Choice," working paper, Kellogg School of Management, Northwestern University, Evanston, IL 60208.

Chernev, Alexander and Ryan Hamilton (2005), "Too Much of a Good Thing? Option Attractiveness and Assortment Choice," working paper, Kellogg School of Management, Northwestern University, Evanston, IL 60208.

Dhar, Ravi (1997), "Consumer Preference for a No-Choice Option," Journal of Consumer Research, 24 (September), 215-31.

Ericsson, Karl A. and Herbert A. Simon (1980), "Verbal Reports as Data," Psychological Review, 87 (July), 215-51.

Greenleaf, Eric A. and Donald R. Lehmann (1995), "Reasons for Substantial Delay in Consumer Decision Making," Journal of Consumer Research, 22 (September), 186-99.

Hauser, John R. and Birger Wernerfelt (1990), "An Evaluation Cost Model of Consideration Sets," Journal of Consumer Research, 16 (March), 393-408.

Hawkins, Scott A. and Stephen J. Hoch (1992), "Low-Involvement Learning: Memory without Evaluation," Journal of Consumer Research, 19 (September), 212-25.

Huffman, Cynthia and Barbara E. Kahn (1998), "Variety for Sale: Mass Customization or Mass Confusion?" Journal of Retailing, 74 (4), 491-513.

Iyengar, Sheena S. and Mark R. Lepper (2000), "When Choice Is Demotivating: Can One Desire Too Much of a Good Thing?" Journal of Personality and Social Psychology, 79 (6), 995-1006.

Jacoby, Jacob, Donald E. Speller, and Carol A. Kohn (1974), "Brand Choice Behavior as a Function of Information Load," Journal of Marketing Research, 11 (February), 63-69.

Kahn, Barbara E. and Donald R. Lehmann (1991), "Modeling Choice among Assortments," Journal of Retailing, 67 (Fall), 274-99.

Kahn, Barbara E., William L. Moore, and Rashi Glazer (1987), "Experiments in Constrained Choice," Journal of Consumer Research, 14 (June), 96-113.

Kahneman, Daniel and Jackie S. Snell (1992), "Predicting a Changing Taste: Do People Know What They Will Like?" Journal of Behavioral Decision Making, 5 (3), 187-200.

Kreps, David M. (1979), "A Representation Theorem for 'Preference for Flexibility,"” Econometrica, 47 (3), 565-77.

Lancaster, Kelvin (1990), "The Economics of Product Variety: A Survey," Marketing Science, 9 (3), 189-206.

Malhotra, Naresh K. (1982), "Information Load and Consumer Decision Making," Journal of Consumer Research, 8 (March), 419-30.

March, James G. (1978), "Bounded Rationality, Ambiguity, and the Engineering of Choice," Bell Journal of Economics, 9 (2), 587-608.

McAlister, Leigh (1982), “A Dynamic Attribute Satiation Model of Variety-Seeking Behavior," Journal of Consumer Research, 9 (September), 141-51.

Payne, John W. (1976), “Task Complexity and Contingent Processing in Decision Making: An Information Search and Protocol Analysis," Organizational Behavior and Human Decision Processes, 16 (2), 366-87.

Ratner, Rebecca K., Barbara E. Kahn, and Daniel Kahneman (1999), "Choosing Less-Preferred Experiences for the Sake of Variety," Journal of Consumer Research, 26 (June), 1-15.

Read, Daniel and George Loewenstein (1995), "Diversification 
Bias: Explaining the Discrepancy in Variety Seeking between Combined and Separated Choices," Journal of Experimental Psychology: Applied, 1 (1), 34-49.

Reibstein, David J., Stuart A. Youngblood, and Howard L. Fromkin (1975), "Number of Choices and Perceived Decision Freedom as a Determinant of Satisfaction and Consumer Behavior," Journal of Applied Psychology, 60 (4), 434-37.

Scammon, Debra L. (1977), "Information Load and Consumers," Journal of Consumer Research, 4 (March), 148-55.

Schwartz, Barry, Andrew Ward, John Monterosso, Sonja Lyubomirsky, Katherine White, and Darrin R. Lehman (2002), "Maximizing versus Satisficing: Happiness Is a Matter of Choice," Journal of Personality and Social Psychology, 83 (5), 1178-97.

Shugan, Steven M. (1980), "The Cost of Thinking," Journal of Consumer Research, 7 (September), 99-111.

Simonson, Itamar (1989), "Choice Based on Reasons: The Case of Attraction and Compromise Effects," Journal of Consumer Research, 16 (September), 158-74.
(1990), "The Effect of Purchase Quantity and Timing on Variety-Seeking Behavior," Journal of Marketing Research, 27 (May), 150-62.

Simonson, Itamar and Stephen M. Nowlis (2000), "The Role of Explanations and Need for Uniqueness in Consumer Decision Making: Unconventional Choices Based on Reasons," Journal of Consumer Research, 27 (June), 49-68.

Simonson, Itamar and Amos Tversky (1992), "Choice in Context: Tradeoff Contrast and Extremeness Aversion," Journal of Marketing Research, 29 (August), 281-95.

Sood, Sanjay, Yuval Rottenstreich, and Lyle Brenner (2004), "On Decisions That Lead to Decisions: Direct and Derived Evaluations of Preference," Journal of Consumer Research, 31 (June), 17-25.

Tversky, Amos and Shmuel Sattath (1979), "Preference Trees," Psychological Review, 86 (6), 542-73.

Tversky, Amos, Shmuel Sattath, and Paul Slovic (1988), "Contingent Weighting in Judgment and Choice," Psychological Review, 95 (July), 371-84. 